\title{
A atuação do enfermeiro na consulta de enfermagem ao paciente hematológico: um relato de experiência
}

\author{
The role of nurses in nursing consultation with hematological patients: an experience report \\ El papel de la enfermera en la consulta de enfermería al paciente hematológico: un relato de \\ experiência
}

Thalyta Mayssa Paiva das Neves

ORCID: https://orcid.org/0000-0002-9960-7309 Universidade do Estado do Pará, Brasil

E-mail: thatamayssa@yahoo.com.br

Ana Caroline Guedes Souza Martins

ORCID: https://orcid.org/0000-0001-7185-8520 Universidade do Estado do Pará, Brasil E-mail: carolguedes.devs@hotmail.com

Wesley Brandão Dias

ORCID: https://orcid.org/0000-0001-5033-9844 Universidade do Estado do Pará, Brasil E-mail:wesleybrandao437@gmail.com

Maiza Silva de Sousa

ORCID: https://orcid.org/0000-0003-3816-8389 Universidade do Estado do Pará, Brasil E-mail: maizasousa1619@gmail.com

Gisele Maria Cardoso da Silva ORCID: https://orcid.org/0000-0003-4202-2913 Universidade Federal do Pará, Brasil E-mail: gcardosomelo@gmail.br

\begin{abstract}
Resumo
Descrever as atividades desenvolvidas pelo enfermeiro na Consulta de Enfermagem (CE) ao paciente hematológico e explicar como a assistência de enfermagem tem sido desenvolvida em um hemocentro. Trata-se de estudo descritivo, do tipo relato de experiência, sobre a atuação do enfermeiro na CE aos pacientes hematológicos em um hemocentro regional do Estado do Pará, atendidos no período de junho a novembro de 2020. A CE foi implantada no local em 2018 e inicialmente, voltou-se apenas para usuários hemofílicos. Esse procedimento está inserido nos programas/ações do Plano Plurianual 2020-2023 “Desenvolvimento Pro Pará. Pra todo o Pará”, sendo contemplado por uma ação que visa, entre outros fatores, a implementação da assistência hematológica integral, com ações de descentralização e abordagem multidisciplinar. Ao chegar pela primeira vez no hemocentro, o usuário é acolhido pela equipe de enfermagem, a qual realiza os primeiros atendimentos. A doença falciforme e a hemofilia A são as doenças hematológicas mais frequentes nos usuários atendidos e quanto às condutas, há predomínio de encaminhamento do usuário para a Prioridade de Atendimento Hematológico, seguido pelo agendamento médico de consultas subsequentes. Desde a sua implantação, algumas conquistas foram alcançadas, porém, atualmente algumas fragilidades têm interferido no aperfeiçoamento da CE, como a falta de uma sistematização consolidada, estudos que evidenciem cientificamente sua relevância para a assistência desses pacientes e a pouca valorização do procedimento pelos usuários, familiares e até pela própria instituição.
\end{abstract}

Palavras-chave: Serviço de hemoterapia; Enfermagem no consultório; Hematologia; Enfermagem.

\begin{abstract}
Describe the activities developed by nurses in the Nursing Consultation (NC) to hematological patients and to explain how nursing care has been developed in a blood center. This is a descriptive study, of an experience report, about the role of nurses in the NC to hematological patients in a regional blood center in the State of Pará, attended from June to November 2020. The NC was deployed on the site in 2018 and bol, turned only to hemophiliac users. This procedure is inserted in the programs / actions of the Pluriannual Plan 2020-2023 "Pro Pará Development. For all Pará", being contemplated by an action that aims, among other factors, the implementation of integral hematological assistance, with decentralization and approach actions multidisciplinary. Upon arriving at the blood center for the first time, the user is welcomed by the nursing team, which performs the first appointments. Sickle cell disease and hemophilia These are the most frequent hematological diseases in the users seen and as for the behaviors, there is a predominance of referral of the user to the Hematological Care Priority, followed by the medical scheduling of subsequent consultations. Since its implementation, some achievements have been achieved, however, currently some weaknesses
\end{abstract}


have interfered in the improvement of the NC, such as the lack of a consolidated systematization, studies that scientifically evidence SUAS, family members and even by the institution itself.

Keywords: Hemotherapy sevice; Office nursing; Hematology; Nursing.

\begin{abstract}
Resumen
Describir las actividades que desarrollan los enfermeros en la Consulta de Enfermería (CE) a los pacientes hematológicos y explicar cómo se ha desarrollado el cuidado de enfermería en un centro de sangre. Se trata de un estudio descriptivo, de un relato de experiencia, sobre el rol de las enfermeras en la $\mathrm{CE}$ a los pacientes hematológicos en un centro regional de sangre en el Estado de Pará, atendido de junio a noviembre de 2020. Se desplegó la CE en el sitio en 2018 e inicialmente, estaba dirigido solo a usuarios hemofílicos. Este procedimiento se inserta en los programas / acciones del Plan Plurianual 2020-2023 "Desarrollo Pro Pará. Para todo Pará", siendo contemplado por una acción que tiene como objetivo, entre otros factores, la implementación de la asistencia hematológica integral, con acciones de descentralización y abordaje. multidisciplinario. Al llegar al centro de sangre por primera vez, el usuario es recibido por el equipo de enfermería, que realiza las primeras citas. La anemia drepanocítica y la hemofilia A son las enfermedades hematológicas más frecuentes en los usuarios atendidos y en cuanto a las conductas, predomina la derivación del usuario a la Prioridad de Atención Hematológica, seguida de la programación médica de consultas posteriores. Desde su implementación se han logrado algunos logros, sin embargo, en la actualidad algunas debilidades han interferido en la mejora de la CE, como la falta de una sistematización consolidada, estudios que demuestran científicamente su relevancia para la atención de estos pacientes y la baja valoración del trámite por usuarios, familiares e incluso por la propia institución.
\end{abstract}

Palabras clave: Servicio de Hemoterapia; Enfermería de oficina; Hematología; Enfermería.

\title{
1. Introdução
}

No Brasil, a Consulta de Enfermagem (CE) é uma prática que vem sendo incorporada nas instituições de saúde de forma gradual, tendo início na década de 60. Sua legalização ocorreu no ano de 1986 com a regulamentação do exercício profissional em enfermagem, por meio da Lei $\mathrm{n}^{\circ}$ 7.498, a qual definiu essa prática como uma atividade privativa do enfermeiro (Brasil, 1986). A CE é uma ferramenta essencial para a assistência de enfermagem, pois caracteriza-se como uma tecnologia relacional que auxilia no estabelecimento de vínculo entre profissional e usuário e por meio da qual o enfermeiro pode prestar uma assistência humanizada voltada à melhoria da sua qualidade de vida (Dantas, Santos \& Tourinho, 2016).

Para o desenvolvimento de uma CE direcionada às necessidades do usuário, torna-se imprescindível a sistematização e organização da prática de cuidado. Nesse sentido, em 2009 o Conselho Federal de Enfermagem (COFEN) divulgou a Resolução no 358 $\square 2009$ que dispõe sobre a Sistematização da Assistência de Enfermagem (SAE) em todos os ambientes de atuação da categoria profissional, a qual se baseia em cinco etapas: coleta de dados ou histórico de enfermagem, diagnóstico, planejamento, implementação e avaliação de enfermagem (COFEN, 2009). Nesse contexto, a CE é uma estratégia tecnológica de cuidado necessária para uma assistência resolutiva, a qual deve estar ancorada em teorias e processos de trabalho sistematizado, afim de promover a saúde, estabelecer o diagnóstico e o tratamento precoce, além de prevenir situações evitáveis (Dantas et al., 2016).

A atuação do enfermeiro em hemoterapia é uma prática antiga, porém, os marcos legais que norteiam essa prática remontam a 1997, quando foi aprovado pelo Conselho Federal de Enfermagem o regulamento sobre a atuação dos profissionais de enfermagem em hemoterapia e transplante de medula óssea. Desde então, essas normas sofreram atualizações e atualmente a atuação do enfermeiro no serviço de hemoterapia é regulamentado pela Resolução COFEN 511/16 (Frantz, 2018). Esta norma técnica dispõe sobre as diretrizes que orientam o trabalho de enfermeiros e técnicos de enfermagem nos serviços de hemoterapia, com o objetivo de qualificar e assegurar uma assistência segura e resolutiva.

As doenças hematológicas caracterizam-se por uma série de distúrbios que afetam os elementos constitutivos do sangue e estão relacionadas tanto a alteração quanto a deficiência de alguns desses fatores, sendo conhecidas como coagulopatias e hemoglobinopatias. As coagulopatias hereditárias, das quais a hemofilia é a mais frequente, estão associadas a deficiência quantitativa e/ou qualitativa de um ou mais fatores de coagulação (proteínas plasmáticas). Pacientes portadores dessas doenças podem apresentar episódios hemorrágicos de gravidade variável, com manifestação espontânea ou pós- 
traumática, que podem estar presentes ao nascer ou serem diagnosticados eventualmente (Brasil, 2015a). A doença de von Willebrand e as hemofilias A e B são os tipos mais comuns, correspondendo a 95\% de todos os casos (Brasil, 2015b).

Enquanto hemoglobinopatia é um termo que abrange uma variedade de patologias caracterizadas pelo desenvolvimento de alterações estruturais e funcionais na hemoglobina, proteína responsável pelo transporte de oxigênio dos pulmões para os tecidos e órgãos, como a doença falciforme e as talassemias (Rosenfeld et al., 2019). As hemoglobinopatias mais comuns são a doença de células falciformes e as talassemias, dependentes, em grande parte, dos procedimentos especializados de hemoterapia (Rosenfeld et al., 2019; Cruz \& Antunes, 2018).

Além das manifestações clínicas, a doença falciforme tem repercussão em vários aspectos da vida da, como interação social, relações conjugais e familiares, educação e emprego. Estudo conduzido por Freitas et al. (2018) sobre a qualidade de vida dos pacientes com essa doença, identificou maior comprometimento quando comparado a população geral em relação ao trabalho e remuneração. Além disso, constatou-se a presença de sinais e sintomas de depressão, ansiedade, abuso de álcool, dor e angústia psicológica, como fatores que contribuem para a diminuição da qualidade de vida dos pacientes portadores de doença falciforme (Freitas et al., 2018).

Dessa forma, percebe-se que os pacientes hematológicos apresentam problemas específicos e em faces destes exige-se um plano de cuidados próprio para este tipo de paciente. Dessa forma, cabe à enfermagem utilizar recursos para aprimorar a assistência prestada a esses pacientes, com implementação de intervenções de enfermagem precisa, que possibilitem a melhoria da qualidade dos resultados esperados. Portanto, é indispensável o preparo da enfermeira, bem como dos familiares que participam do processo de assistência ao portador de alterações hematológicas (Lopes \& Gomes, 2020).

No entanto, uma busca na literatura sobre a utilização da CE para assistência à pacientes hematológicos encontrou apenas um estudo, realizado por Oliveira, Silva e Monteiro (2020) em um Hemocentro Regional de Pouso Alegre. Isso, de certa forma, traz uma limitação para o presente relato e por outro lado, remonta a importância de abordagem do tema e desperta o interesse para a sua investigação, uma vez que ainda não foi explorado e torna-se cada vez maior o número de usuários que precisam de uma assistência de enfermagem direcionada às suas necessidades.

Nesse contexto, busca-se a construção de conhecimentos específicos que deem suporte na definição e melhoria na qualidade de assistência ao usuário através da descrição da atuação do enfermeiro na CE em um hemocentro regional.

\section{Metodologia}

Trata-se de um estudo descritivo, do tipo relato de experiência, sobre a atuação do enfermeiro na CE aos pacientes hematológicos de um hemocentro regional do Estado do Pará e tem como objetivo descrever as atividades desenvolvidas pelo enfermeiro na CE ao paciente hematológico e explicar como a assistência de enfermagem tem sido desenvolvida no local, apontando as potencialidades e fragilidades observadas.

O método descritivo visa descrever as características conhecidas ou componentes do fato, fenômeno ou representação. Este método possibilita o desenvolvimento de um nível de análise em que é possível identificar as diferentes formas do fenômeno, sua ordenação e classificação. Dá margem também a explicação das relações de causa e efeito dos fenômenos, proporcionando ao pesquisador a obtenção de melhor compreensão do comportamento dos diferentes fatores e elementos que influenciam determinado fenômeno (Freire \& Pasttussi, 2018).

$\mathrm{O}$ relato de experiência é uma modalidade de pesquisa qualitativa em que o indivíduo revisita fatos e memórias que tenham um significado próprio e constrói um direcionamento de pesquisa. Assim, o relato de experiência vai além da mera descrição de fatos e eventos, ele propõe uma explicitação descritiva com base na interpretação e compreensão dos fenômenos ocorridos em um dado momento (Daltro \& Faria, 2019). Dessa forma, o relato é feito de modo contextualizado, com objetividade e aporte teórico. 
Desta feita, o presente estudo trata das experiências vivenciadas por uma enfermeira em um hemocentro regional do Estado do Pará, no período de junho a novembro de 2020. Este está vinculado ao hemocentro coordenador da Fundação Centro de Hemoterapia e Hematologia do Pará (HEMOPA) e atende as demandas de 47 municípios do estado.

O hemocentro conta com uma equipe multiprofissional distribuída entre enfermeiros, médicos, assistentes sociais, farmacêuticos, técnicos em enfermagem, técnicos em hematologia e agentes administrativos. Os serviços oferecidos se classificam em complexidade intermediária, com o desenvolvimento de atividades como: a captação e o processamento de sangue, testes sorológicos, o diagnóstico e acompanhamento de pacientes com doenças hematológicas, a estocagem e distribuição de hemocomponentes e hemoderivados.

Além disso, o local é referência na região para o acompanhamento de pacientes hematológicos, possui um ambulatório com funcionamento de segunda a sexta-feira, das 07:00 às 13:00 horas. Os atendimentos acontecem de forma agendada ou por demanda espontânea. O agendamento pode ser feito presencialmente ou por contato telefônico, no caso de usuários pertencentes a municípios vizinhos. No dia do atendimento os usuários precisam atender aos seguintes critérios quanto à apresentação de documentação: apresentar a guia de referência-contra referência, cartão do SUS, registro de identificação e comprovante de residência.

\section{Resultados}

Durante a descrição desse estudo serão mencionados os atendimentos realizados aos pacientes portadores de doenças hematológicas, incluído os usuários em acompanhamento por coagulopatias e hemoglobinopatias, que totalizam 69 pacientes, de acordo com informações da instituição. No local, os pacientes recebem atendimento de uma equipe multiprofissional, que é formada por médicos, enfermeiros, assistentes sociais, farmacêuticos, técnicos de enfermagem e técnicos de hematologia.

A equipe de enfermagem que presta assistência na instituição é constituída por 11 (onze) profissionais, sendo 05 (cinco) enfermeiros e 06 (seis) técnicos de enfermagem. Entre as atividades desenvolvidas pelo enfermeiro no ciclo do sangue estão: captação de doadores, triagem clínica, coleta de sangue doado, distribuição do sangue, hemovigilância e atividades assistenciais desenvolvidas ao paciente hematológico que incluem, entre outras, supervisão na administração de sangue e hemoderivados, acolhimento ao paciente e CE.

A CE foi implantada no local em 2018, motivado pelo serviço que estava sendo realizado no hemocentro coordenador na capital do Estado e para atender aos protocolos ministeriais e programas de profilaxia primária, profilaxia secundária e imunotolerância desenvolvido para pessoas portadoras de coagulopatias hereditárias. Foram desenvolvidas capacitações teórico-práticas com uma enfermeira atuante no serviço, em relação aos protocolos gerais existentes e a atuação específica do enfermeiro no serviço.

Inicialmente, a CE foi voltada apenas para usuários hemofílicos, sendo, posteriormente, ampliada aos portadores de hemoglobinopatias e durante o período da vivência, o atendimento também foi estendido aos demais usuários portadores de doenças hematológicas. E no período estudado, o consultório de enfermagem passou por adaptações e as consultas, que inicialmente eram realizadas em uma sala compartilhada, ganharam um novo espaço, com um consultório específico. Apesar de algumas limitações, essa conquista de espaço demonstra reconhecimento e valorização pelo trabalho que vem sendo desenvolvido pela enfermagem na instituição, frente às demandas dos pacientes.

A CE está inserida nos programas/ações do Plano Plurianual 2020-2023 "Desenvolvimento Pro Pará. Pra todo o Pará", que é um instrumento de planejamento que tem o objetivo de nortear uma gestão transparente na execução dos programas/ações que viabilizam a oferta de produtos e serviços de qualidade, como resposta as necessidades declaradas da sociedade. No referido plano, a ação que contempla a CE é denominada Implementação dos Serviços de Média e Alta complexidade, que visa a implementação da assistência hematológica integral, com ações de descentralização em conjunto 
com a equipe multidisciplinar, além da adequação do ambulatório de pacientes, com foco no atendimento multidisciplinar, multiprofissional e humanizado.

O procedimento de CE foi incluso na Programação Físico Orçamentária (FPO) da instituição a partir do mês de julho de 2020 e está classificado como consulta de outros profissionais de nível superior, com a média de 14 atendimentos ao mês. Uma grande conquista para a instituição e para a enfermagem foi ter o seu atendimento contabilizado não somente de forma estatística e burocrática, mas também reconhecida e com visibilidade no setor financeiro. A meta física, programada para o ano de 2020, referente a esse procedimento no hemocentro é de 150 atendimentos ao ano. Entre os atendimentos de enfermagem prevalecem a assistência ao usuário com hemoglobinopatias e coagulopatias.

Ao chegar pela primeira vez no hemocentro por demanda espontânea, o usuário é acolhido pela equipe de enfermagem, a qual realiza os primeiros atendimentos, por meio de escuta qualificada e exame físico, com aferição de sinais vitais, temperatura corporal, pressão arterial, peso, altura e dosagem de hemoglobina. Durante essa avaliação inicial é identificado principalmente se o usuário é perfil do hemocentro, se a condição atual de saúde exige prioridade no atendimento médico e abrem-se oportunidades para o esclarecimento de dúvidas e orientações gerais.

Estabelecido esse primeiro contato, é colhido o histórico de enfermagem e realizado o exame físico completo e específico. Inclui-se no prontuário do paciente a ficha de evolução de enfermagem e de acordo com o seu diagnóstico, se adota um instrumento padrão específico para pacientes com hemoglobinopatias e outro para coagulopatias, elaborado pelo hemocentro coordenador. Ainda não é desenvolvido o processo de enfermagem durante os atendimentos, porém as orientações de enfermagem seguem as recomendações estabelecidas para atender as necessidades do usuário de acordo com suas particularidades.

Os usuários que possuem diagnóstico de hemoglobinopatias (talassemia, doença falciforme) ou coagulopatias (hemofilia, doença de Von Willebrand) são acompanhados pela equipe multidisciplinar da instituição. Os atendimentos de enfermagem a esses usuários são conduzidos através da utilização dos Procedimentos Operacionais Padrões (POPs), estabelecidos pelo hemocentro coordenador e pelos programas ministeriais. Estes protocolos e programas são criados especificamente para atender as particularidades do paciente com coagulopatias e hemoglobinopatias durante a CE.

Durante a vivência, verificou-se que a doença falciforme e a hemofilia A são as doenças hematológicas mais frequentes nos usuários atendidos durante as consultas de enfermagem. E quanto às condutas, nos desfechos das consultas de enfermagem, há predomínio de encaminhamento do usuário para a Prioridade de Atendimento Hematológico (PAH), seguido pelo agendamento médico das consultas subsequentes. O PAH é um serviço disponível no hemocentro regional para usuários que precisam de prioridade no atendimento em virtude da gravidade de seu quadro clínico como: presença de equimoses, sangramentos, dores, febre, crises álgicas, níveis de hemoglobina muito abaixo ou muito acima do normal, além de outras referências alteradas nos padrões do hemograma.

Além das características da CE já descritas anteriormente, faz-se necessário mencionar as demais competências do enfermeiro atuante na $\mathrm{CE}$, como as atividades técnicas educativas, incluindo a participação nas reuniões técnicas, oficinas, visita domiciliar e treinamento da dose domiciliar (treinamento de auto infusão destinado ao familiar e usuário que tem indicação de administração do fator de coagulação em domicílio). É importante elucidar que no período estudado as atividades técnicas educativas foram suspensas por conta da pandemia do novo coronavírus.

\section{Discussão}

A CE é uma estratégia eficaz para a detecção precoce de desvios de saúde e acompanhamento de medidas instituídas, as quais se dirigem ao bem-estar das pessoas. E sua sistematização torna-se essencial para organizar o cuidado e oferecer uma assistência integral (Barros et al., 2015). Nesse sentido, uma iniciativa extremamente importante para melhorar a assistência é a 
implantação da SAE, instituída pela Resolução COFEN n 358/2009, a qual dispõe sobre a sua implementação em todos os ambientes de atuação do enfermeiro (COFEN, 2009). Nesse contexto, a CE é uma estratégia tecnológica de cuidado necessária para uma assistência resolutiva, a qual deve estar ancorada em teorias e processos de trabalho sistematizado, afim de promover a saúde, estabelecer o diagnóstico e o tratamento precoce, além de prevenir situações evitáveis (Dantas et al., 2016).

As consultas de enfermagem realizadas no hemocentro ainda não utilizam todas as etapas da SAE, fato que merece ser estimulado aos profissionais e pesquisadores, uma vez que é preciso estudar a possibilidade de adoção e inclusão do Diagnóstico de Enfermagem nos atendimentos ao paciente hematológico. Mas apesar da SAE não estar implantada no local, são utilizados instrumentos fornecidos pelo hemocentro coordenador, os quais orientam os procedimentos durante a CE e estão voltados às demandas dos usuários, procurando atender suas particularidades.

A utilização desses instrumentos constituem uma importante iniciativa para sistematizar a assistência de enfermagem no local, sendo necessário a articulação da equipe e coordenação para a efetivação dessa ferramenta, uma vez que sua utilização é essencial para melhorar a comunicação entre a equipe, o processo de trabalho, bem como a assistência de enfermagem a cada usuário (Barros et al., 2015).

Um estudo que reflete sobre a utilização da SAE em ambulatórios de hemofilia no estado do Ceará, avaliou o conhecimento de enfermeiras sobre esse instrumento, identificando que $87,5 \%$ das entrevistadas conhecem todas as etapas do Processo de Enfermagem (PE), mas a SAE ainda não foi implantada nos serviços em que atuam. A pesquisa ressalta, ainda, que apenas algumas etapas do PE, como a coleta de dados e intervenções são realizadas nos ambulatórios (Souza et al., 2016).

Além desses fatores, outro aspecto extremamente relevante para uma assistência de qualidade é o acolhimento do usuário. Rodrigues et al. (2020), ao relatar a experiência sobre a utilização de tecnologias em saúde na formação de gestores, aborda a importância do conhecimento dessas tecnologias para o desenvolvimento de uma assistência baseada na escuta ativa, com melhoria da recepção e atendimento dos usuários que chegam ao serviço.

É importante mencionar que a CE traz grande impacto nos modos de produzir saúde, no envolvimento das famílias no processo de cuidado do indivíduo e na atenção prestada. Além disso, é preciso que o enfermeiro atente ao gerenciamento de seus múltiplos papéis, para que não se exima da sua responsabilidade de cuidador e acabe por assumir funções meramente administrativas ou medicalizantes. Entende-se que essas funções devem coexistir com o manejo das respostas do indivíduo aos agravos de saúde e com a capacidade de uma abordagem sociocultural específica de cada indivíduo compreendido dentro de um processo familiar. Todos esses fatores devem atuar simultaneamente no exercício da CE (Amaral \& Abrahão, 2017).

A procura pelo atendimento de enfermagem pelos pacientes portadores de hemoglobinopatias, como a doença falciforme apresentou-se mais frequente que os demais pacientes de outros diagnósticos. E no momento do atendimento é importante se atentar para os fatores que vão além das manifestações clínicas próprias da doença, como a presença de sinais e sintomas de depressão, ansiedade, abuso de álcool, dor e angústia psicológica, pois são fatores que contribuem para a diminuição da qualidade de vida dos pacientes portadores de doença falciforme (Freitas et al., 2018).

Durante o período de vivência na instituição foi perceptível que a CE, tanto para a instituição quanto para o paciente, precisa ser divulgada e esclarecida. Muitos ainda percebem apenas como uma triagem ou uma etapa do protocolo que precisa ser seguido. Faz-se necessário o desenvolvimento de pesquisas sobre a importância das atividades de enfermagem para o processo de cuidado do usuário hematológico, o significado da $\mathrm{CE}$ ao paciente e familiar de pessoas com essas doenças, assim como ratificar o fluxo de atendimento da enfermagem no hemocentro.

Estes achados somados com o estudo de Amaral et al. (2016), afirmam que embora o enfermeiro seja cada vez mais requisitado nas diversas atividades ligadas à hematologia, não existe reconhecimento por parte da própria categoria, pelo fato de que esses profissionais desconhecem a complexidade do serviço. É possível que tal desconhecimento ocorra, em grande parte, em razão de o conteúdo do curso de graduação em enfermagem não enfatizar tal abordagem ou o conteúdo ser 
insuficiente em sua grade curricular, além de haver um reduzido número de cursos de pós-graduação lato sensu voltados para essa área.

Além disso, é importante destacar que no contexto da assistência de enfermagem aos pacientes, esses profissionais desenvolvem uma série de atividades, desde cuidados preventivos, até cuidados educativos, perpassando cuidados clínicos que requerem um manejo adequado as necessidades do paciente, como mostra o estudo de Sousa, Santo e Pinheiro, 2017.

Com isso, destaca-se a necessidade de sensibilização das pessoas para a valorização das atividades desenvolvidas pelo profissional enfermeiro, de forma que as consultas possam trazer maiores contribuições aos usuários e familiares, pois trata-se de um instrumento efetivo de prevenção de problemas de saúde, que é eficaz, porém, pouco explorado (Pereira \& Ferreira, 2014). Ademais, a necessidade de abordagem do tema se tornou evidente ao longo desta pesquisa pela escassez de publicações que retratam a CE diretamente ao paciente hematológico.

\section{Considerações Finais}

Os usuários portadores de doenças hematológicas apresentam diversas demandas de cuidado que requerem manejo por profissionais capacitados. No hemocentro regional em que vivenciei essa experiência, a CE é um procedimento consolidado na rotina de atendimento a esses pacientes e que contribui significativamente no seu processo de cuidado. Desde a sua implantação, em 2018, algumas conquistas foram alcançadas, porém, atualmente algumas fragilidades têm interferido no seu aperfeiçoamento, como a falta de uma sistematização consolidada, estudos que evidenciem cientificamente a relevância da CE para a assistência desses pacientes e a pouca valorização do procedimento pelos usuários, familiares e até a própria instituição.

Portanto, acredita-se que os enfermeiros exercem um papel fundamental no atendimento ao paciente portador de doenças hematológicas e precisam estar adequadamente preparados para desempenhar esta responsabilidade, buscando a redução das distâncias entre a prática e o conhecimento científico disponível, de forma a ofertar um atendimento sistematizado e consequentemente, de qualidade. Sugere-se a realização de novos estudos sobre o trabalho do enfermeiro em serviços de hemoterapia quanto à realização da $\mathrm{CE}$, tendo em vista o seu papel educativo para a população e equipe de enfermagem.

Apesar das publicações estarem aumentando nos últimos anos no que se refere à hemoterapia, é necessário à realização de pesquisas na enfermagem que incentivem a discussão nessa área do conhecimento, principalmente quanto à sistematização da assistência. Acredita-se que o desenvolvimento científico na área contribuirá para a consolidação da profissão de enfermagem, ajudando-a alcançar o status e a valorização profissional, frente à sociedade e as demais profissões.

\section{Referências}

Amaral, I. T. \& Abrahão, A. L. (2017). Consulta em enfermagem na Estratégia Saúde da Família, ampliando o reconhecimento das distintas formas de ação: uma revisão integrativa. Revista Cuidado é Fundamental, 9(4), 899-906. https://pesquisa.bvsalud.org/portal/resource/pt/biblio-908484.

Amaral, J. H. S. et al. (2016). Hemoterapia: um desafio no cotidiano da equipe de enfermagem. Revista Enfermagem UFPE online, 10 (6), $4820-7$. https://periodicos.ufpe.br/revistas/revistaenfermagem/article/view/11261.

Barros, A. L. B. L. et al. (2015). Processo de enfermagem. In: Conselho Regional de Enfermagem de São Paulo, Processo de Enfermagem: Guia para a Prática, pp 36-54. COREN.

Brasil. (1986). Lei n. 7.498, de 25 de junho de 1986. Dispõe sobre a regulamentação do exercício da Enfermagem e dá outras providências. http://www.cofen.gov.br/lei-n-749886-de-25-de-junho-de-1986_4161.html.

Conselho Federal de Enfermagem. (2009). Resolução $n^{\circ}$ 358/2009, de 15 de outubro de 2009. Dispõe sobre a Sistematização da Assistência de Enfermagem e a implementação do Processo de Enfermagem em ambientes, públicos ou privados, em que ocorre o cuidado profis sional de Enfermagem, e dá outras providências. http://www.cofen.gov.br/resoluo-cofen-3582009_4384.html.

Cruz, T. C. \& Antunes, L. (2018). Fisiopatogenia e métodos diagnósticos das anemias hemolíticas: uma revisão integrativa. Revista Brasileira de Crescimento e Desenvolvimento Humano, 6 (2) 49-61. https://revistas.unilasalle.edu.br/index.php/saude_desenvolvimento/article/view/4259. 
Research, Society and Development, v. 10, n. 4, e15810413916, 2021

(CC BY 4.0) | ISSN 2525-3409 | DOI: http://dx.doi.org/10.33448/rsd-v10i4.13916

Daltro, M. R. \& Faria, A.A. (2019). Relato de experiência: Uma narrativa científica na pós-modernidade. Estudos e Pesquisas em Psicologia, 19 (1), $223-237$. https://www.e-publicacoes.uerj.br/index.php/revispsi/article/view/43015.

Dantas, C. N., Santos, V. E. P. \& Tourinho, F. S. V. (2016). A consulta de enfermagem como tecnologia do cuidado à luz dos pensamentos de Bacon e Galimberti. Texto Contexto Enfermagem, 25(1), e2800014. http://dx.doi.org/10.1590/0104-0707201500002800014.

Frantz, S. R. S. (2018). Processo de trabalho dos enfermeiros no Serviço de hemoterapia: entre o prescrito e o real. (Tese de doutorado). Universidade Federal de Santa Catarina, Florianópolis, SC, Brasil. https://repositorio.ufsc.br/handle/123456789/205575.

Freire, M. C. M. \& Pasttussi, M.P. (2018). Princípios da Pesquisa. Tipos de estudo. In: Estrela, C. Metodologia científica: ciência, ensino, pesquisa. (3a ed., pp. 109-125). Porto Alegre: Artes Médicas.

Freitas, S. L. F, et al. (2018). Qualidade de vida em adultos com doença falciforme: revisão integrativa da literatura. Revista Brasileira de Enfermagem, 71(1),207-17. http://dx.doi.org/ 10.1590/0034-7167-2016-0409.

Lopes, W. S. L. \& Gomes, R. (2020). A participação dos conviventes com a doença falciforme na atenção à saúde: um estudo bibliográfico. Ciência e Saúde Coletiva, 25(8), 3239-3250. https://www.scielo.br/scielo.php?script=sci_arttext\&pid=S1413-81232020000803239\&lng=es\&nrm=iso.

Ministério da Saúde. (2015a). Manual de hemofilia. Brasília: Ministério da Saúde. http://biblioteca.cofen.gov.br/wpcontent/uploads/2016/03/manual_hemofilia_2ed.pdf.

Ministério da Saúde. (2015b). Manual das coagulopatias hereditárias raras. Brasília: Ministério da Saúde. http://bvsms.saude.gov.br/bvs/publicacoes/manual_coagulopatias_hereditarias_raras.pdf.

Oliveira, C. E., Silva, R. P. \& Monteiro, F. P. (2020). Consulta de enfermagem aos pacientes portadores de coagulopatias hereditárias do Hemocentro Regional de Pouso Alegre - Fundação Hemominas: relato de experiência. Hematology, transfusion and cell therapy, 42(2), 457-458. http://www.htct.com.br/en-consulta-de-enfermagem-aos-pacientes-articulo-S2531137920310567.

Pereira, R. T. A. \& Ferreira, V. (2014). A Consulta de enfermagem na estratégia saúde da família. Revista Brasileira Multidisciplinar, 17(1), 99-111. https://revistarebram.com/index.php/revistauniara/article/view/10.

Rodrigues, R. P. et al. (2020). Tecnologias em Saúde: Aperfeiçoar o Processo de Trabalho Pautado na Gestão da Clínica e do Cuidado. Brazilian Journal of Development, 6(1), 2922- 2932. https://www.brazilianjournals.com/index.php/BRJD/article/view/6221/5523.

Rosenfeld, L. G. et al. (2019). Prevalência de hemoglobinopatias na população adulta brasileira: Pesquisa Nacional de Saúde 2014-2015. Revista Brasileira de Epidemiologia, 22 (2), E190007. 10.1590/1980-549720190007.supl.2

Sousa, R. M., Espírito Santo, F. H. \& Pinheiro, F. (2017). Tipologia do cuidado de enfermagem ao paciente onco-hematológico: um estudo de caso. Revista Enfermagem Atual, edição Especial, 81-86. http://revistaenfermagematual.com.br/index.php/revista/article/view/554.

Souza, V. N. E. et al. (2016). Conhecimento das enfermeiras de ambulatórios de hemofilia sobre a sistematização da assistência de enfermagem. Revista de Enfermagem UFPE on line, 10(5),1654-62. 10.5205/reuol.9003-78704-1-SM.1005201611. 